\title{
Letter
}

\section{Internalizing Conservation through Our Own Microbes}

Emerging links between the microbes that inhabit our own bodies and our health promise to revolutionize the way we perceive ecology and conservation. Redford et al. (2012 [this issue]) rightly argue that we should link the issue of the human microbiome and health to the broader conservation of species. These microbial communities are the product of 3.5 billion years of evolution and perceive macrospecies as complex environments that they alter and to which they adapt. Assessing their fundamental role in conservation and ecosystem function should also consider the scale at which differences in microbial communities are important. Within the human body, medical microbiologists are revisiting links between community ecology and immune function and inoculating of patients with an entire microbial community from a healthy individual to help cure localized diseases (Brandt et al. 2011). As Redford et al. argue, the informed management of microbes is also likely to stabilize health of individual animals or populations in zoos and may also be an effective tool in restoring entire ecosystems (Harris 2009; Kardol \& Wardle 2010). The human microbiome in particular offers an unusual opportunity to allow people to understand why conservation science is so important.

Our bodies' microbial communities are filled with a diverse array of species and are affected by invasions and disturbances, all of which fundamentally affect the stability of our health. Our bodies and their microbes are direct analogs to the nonhuman ecosystems and species that conservation professionals are struggling to protect and restore. I believe conservation scientists should work diligently to ensure that our language and that of medical microbiologists are parallel in terminology and message. For example, people sickened by bacterial infections of 
the gut have had their gut microbiome invaded, which has affected the homeostasis and function of their stomachs. This kind of event is directly analogous to visible ecosystem invasions, such as lampreys (Petromyzon marinus) in the Great Lakes of the United States and Canada or pine trees in the southern hemisphere. People may be better able to internalize future conservation issues by recognizing the similarities between human health and the health of other ecosystems through microbial communities. Within education, the unification of conservation science and the human microbiome should happen as early as possible. For example, biology students could manipulate easily cultured microbial communities from their own bodies and measure how microbial diversity alters functions such as resource use or resistance to chemical disturbance. These same tests could then be conducted either on microbes from surrounding environments (roots, animals or water) or at the non-microbial scale directly with plants or invertebrates. The resulting juxtaposition of conservation science and human microbiology will increase student interest in finding parallels between conserving and repairing the human ecosystem and conserving and repairing ecosystems in the broader natural world. With such a focus, microbes may help bridge the knowledge gap between humans and the natural world.

Benjamin A. Sikes, University of Texas at Austin, Austin, TX, 78712, USA e-mail bensikes@mail.utexas.edu

\section{Literature Cited}

Brandt, L. J., T. J. Borody, and J. Campbell. 2011. Endoscopic fecal microbiota transplantation:"first-line" treatment for severe Clostridium difficile infection? Journal of Clinical Gastroenterology 45:655.

Harris, J. 2009. Soil microbial communities and restoration ecology: facilitators or followers? Science 325:573-574. 
Kardol, P., and D. A. Wardle. 2010. How understanding aboveground-belowground linkages can assist restoration ecology. Trends in Ecology \& Evolution 25: 670-679. 